\title{
BMJ Open Aspirin and clonidine in non-cardiac surgery: acute kidney injury substudy protocol of the Perioperative Ischaemic Evaluation (POISE) 2 randomised controlled trial
}

Amit X Garg, ${ }^{1}$ Andrea Kurz, ${ }^{2}$ Daniel I Sessler, ${ }^{2}$ Meaghan Cuerden, ${ }^{3}$ Andrea Robinson, ${ }^{4}$ Marko Mrkobrada, ${ }^{1}$ Chirag Parikh, ${ }^{5}$ Richard Mizera, ${ }^{4}$ Philip M Jones, ${ }^{1}$ Maria Tiboni, ${ }^{4}$ Raul Gonzalez Rodriguez, ${ }^{6}$ Ekaterina Popova, ${ }^{6}$ Maria Fernanda Rojas Gomez, ${ }^{7}$ Christian S Meyhoff, ${ }^{8}$ Tomas Vanhelder, ${ }^{4}$ Matthew T V Chan, ${ }^{9}$ David Torres, ${ }^{10}$ Joel Parlow, ${ }^{11}$ Miriam de Nadal Clanchet, ${ }^{12}$ Mohammed Amir, ${ }^{13}$ Seyed Javad Bidgoli, ${ }^{14}$ Laura Pasin, ${ }^{15}$ Kristian Martinsen, ${ }^{16}$ German Malaga, ${ }^{17}$ Paul Myles, ${ }^{18}$ Rey Acedillo, ${ }^{1}$ Pavel Roshanov, ${ }^{1}$ Michael Walsh, ${ }^{4}$ George Dresser, ${ }^{1}$ Priya Kumar, ${ }^{19}$ Edith Fleischmann, ${ }^{20}$ Juan Carlos Villar, ${ }^{21}$ Tom Painter, ${ }^{22}$ Bruce Biccard, ${ }^{23}$ Sergio Bergese, ${ }^{24}$ Sadeesh Srinathan, ${ }^{25}$ Juan P Cata, ${ }^{26}$ Vincent Chan, ${ }^{27}$ Bhupendra Mehra, ${ }^{28}$ Kate Leslie, ${ }^{29}$ Richard Whitlock, ${ }^{4} \mathrm{P}$ J Devereaux, ${ }^{4}$ on behalf of the POISE-2 Investigators

To cite: Garg AX, Kurz A, Sessler DI, et al. Aspirin and clonidine in non-cardiac surgery: acute kidney injury substudy protocol of the Perioperative Ischaemic Evaluation (POISE) 2 randomised controlled trial. BMJ Open 2014:4:e004886. doi:10.1136/bmjopen-2014004886

- Prepublication history and additional material for this paper is available online. To view these files please visit the journal online (http://dx.doi.org/10.1136/ bmjopen-2014-004886).

Received 18 January 2014 Accepted 21 January 2014

CrossMark

For numbered affiliations see end of article.

Correspondence to Dr Amit Garg; amit.garg@|hsc.on.ca

\section{ABSTRACT}

Introduction: Perioperative Ischaemic Evaluation-2 (POISE-2) is an international $2 \times 2$ factorial randomised controlled trial of low-dose aspirin versus placebo and low-dose clonidine versus placebo in patients who undergo non-cardiac surgery. Perioperative aspirin (and possibly clonidine) may reduce the risk of postoperative acute kidney injury (AKI).

Methods and analysis: After receipt of grant funding, serial postoperative serum creatinine measurements began to be recorded in consecutive patients enrolled at substudy participating centres. With respect to the study schedule, the last of over 6500 substudy patients from 82 centres in 21 countries were randomised in December 2013. The authors will use logistic regression to estimate the adjusted OR of AKI following surgery (compared with the preoperative serum creatinine value, a postoperative increase $\geq 26.5 \mu \mathrm{mol} / \mathrm{L}$ in the 2 days following surgery or an increase of $\geq 50 \%$ in the 7 days following surgery) comparing each intervention to placebo, and will report the adjusted relative risk reduction. Alternate definitions of AKI will also be considered, as will the outcome of AKI in subgroups defined by the presence of preoperative chronic kidney disease and preoperative chronic aspirin use. At the time of randomisation, a subpopulation agreed to a single measurement of serum creatinine between 3 and 12 months after surgery, and the authors will examine intervention effects on this outcome.

Ethics and dissemination: The authors were competitively awarded a grant from the Canadian Institutes of Health Research for this POISE-2 AKI

\section{Strengths and limitations of this study}

- International recruitment of over 6500 patients across 82 centres in 21 countries will provide generalisable estimates of the treatment effects on acute kidney injury (AKI).

- The kidney data collection schedule in this large international trial is feasible and efficient.

- Additional studies of the effects of aspirin in the surgical setting can consider multiple measures of kidney function over time, both before and long after AKI, examine long-term trajectories of kidney function loss and new markers of kidney function or injury.

substudy. Ethics approval was obtained for additional kidney data collection in consecutive patients enrolled at participating centres, which first began for patients enrolled after January 2011. In patients who provided consent, the remaining longer term serum creatinine data will be collected throughout 2014. The results of this study will be reported no later than 2015.

Clinical Trial Registration Number: NCT01082874.

\section{BACKGROUND}

Worldwide, an estimate of 200 million adults have major non-cardiac surgery each year. ${ }^{1}$ About $10 \%$ of patients develop acute kidney 
injury (AKI) (defined by an acute rise in serum creatinine of $50 \%$ or more or an acute rise of $26 \mu \mathrm{mol} / \mathrm{L}$ or more), and $0.5 \%$ receive acute dialysis. ${ }^{2}$ In other words, worldwide there are several million annual cases of AKI attributable to major non-cardiac surgery, with about a million cases receiving acute dialysis. When AKI occurs (versus when it does not), it is associated with increased mortality, longer hospital length of stay and higher healthcare costs. ${ }^{3-5}$ Treatments to prevent the onset of AKI or its complications remain elusive. ${ }^{6}$

The major mechanism of perioperative AKI is impaired kidney perfusion and ischaemia. Activation of inflammatory mediators, adhesion molecules, platelets and thromboxane is thought to be involved in the injury (figure 1). ${ }^{78}$

In the surgical setting, aspirin prevents platelet aggregation and may improve glomerular blood flow. In online supplementary appendix 1, we provide three lines of evidence to support the hypothesis that perioperative aspirin use reduces the risk of postoperative AKI. ${ }^{7-15}$ The pathways where aspirin may mitigate AKI are presented in figure $1 .^{16}$

We are conducting the Perioperative Ischaemia Evaluation-2 trial (POISE-2). The methods of this large, international $2 \times 2$ factorial randomised placebo controlled trial are described elsewhere (available from the authors on request). ${ }^{17}$ In brief, adults at moderate and high risk for postoperative cardiac events are randomly allocated to receive either aspirin or matching placebo, and clonidine or matching placebo. Eligible surgeries are those where a patient is expected to stay for at least one night in the hospital after surgery. The aspirin intervention is $100 \mathrm{mg}$ tablets of aspirin or matching placebo, where patients take two tablets orally $2-4 \mathrm{~h}$ prior to surgery, and then take one tablet daily for either 7 or 30 days (depending on whether they were taking aspirin chronically prior to surgery or not, respectively). The clonidine intervention consists of $0.2 \mathrm{mg}$ of oral clonidine or matching placebo given $2-4 \mathrm{~h}$ prior to surgery, and at the same time a transdermal clonidine or placebo patch is applied for $72 \mathrm{~h}$, providing clonidine at $0.2 \mathrm{mg} /$ day. Allocation is performed by a central randomisation service and is stratified by the centre. Patients, healthcare providers, data collectors and outcome adjudicators are blinded to treatment allocation. This trial is primarily funded by the Canadian Institutes of Health Research. With respect to the study schedule, the last of 10000 patients from 140 centres in 23 countries was randomised in December 2013. The primary outcome is a composite of 30 day all-cause mortality or non-fatal myocardial infarction. The secondary outcome is a composite of 30 day all-cause mortality, non-fatal myocardial infarction and non-fatal stroke.

In addition to the primary outcome, POISE-2 is uniquely positioned to determine the effects of perioperative aspirin (and clonidine) on AKI, to consider whether treatment effects on AKI differ in those with preoperative chronic kidney disease (CKD) and preoperative chronic aspirin use, and to investigate the treatment effects on longer term kidney function, based on a single measurement taken 3 months to 1 year after surgery. We were awarded an additional grant from the Canadian Institutes of Health Research to examine these issues in a POISE-2 AKI substudy. The AKI questions detailed in the grant are presented below and are followed by our prespecified analytic plan.

\section{Primary question}

1. In patients undergoing non-cardiac surgery, does the use of aspirin at the time of surgery compared with placebo alter the risk of postoperative AKI? Is the treatment effect (the observed relative risk reduction) similar across alternate definitions of AKI? Hypothesis: perioperative aspirin versus placebo at the time of surgery will reduce the risk of postoperative AKI, and the treatment effect will appear similar across alternate definitions of AKI.

\section{Secondary questions}

2. Does the observed relative risk reduction of aspirin versus placebo on postoperative AKI differ in patients with and without preoperative CKD? Hypothesis: the relative risk reduction will be greater in patients with preoperative CKD than in those without CKD (as we demonstrated in another setting-a large international randomised trial of coronary artery bypass surgery performed either with or without a bypass pump).

3. Does the observed relative risk reduction of aspirin versus placebo on postoperative AKI differ in patients who were chronically taking aspirin before surgery, compared to those who had aspirin initiated at the time of surgery? Hypothesis: the relative risk reduction will be greater in the aspirin continuation stratum than in the aspirin initiation stratum, as there may be an increased risk of thrombosis after aspirin discontinuation (as observed in some human studies, recognising the biology is complicated).$^{18}$

4. Does perioperative aspirin versus placebo alter longer term kidney function (indicated by a single measure of serum creatinine 3-12 months after surgery)? Hypothesis: we will be unable to demonstrate a better outcome with perioperative aspirin versus placebo due to limitations in the measurement techniques, or the limited impact of any observed AKI risk reduction on longer term kidney function.

\section{Other questions}

5. In patients undergoing non-cardiac surgery, does the use of perioperative clonidine at the time of surgery compared with placebo alter the risk of postoperative AKI? Hypothesis: perioperative clonidine versus placebo at the time of surgery will reduce the risk of postoperative AKI (a notion supported by some animal and human studies, ${ }^{19-24}$ recognising that an 
Figure 1 Mechanism for development of acute kidney injury in major non-cardiac surgery: role of aspirin. ACEi, angiotensin converting enzyme inhibitors; ARB, angiotensin receptor blockers.

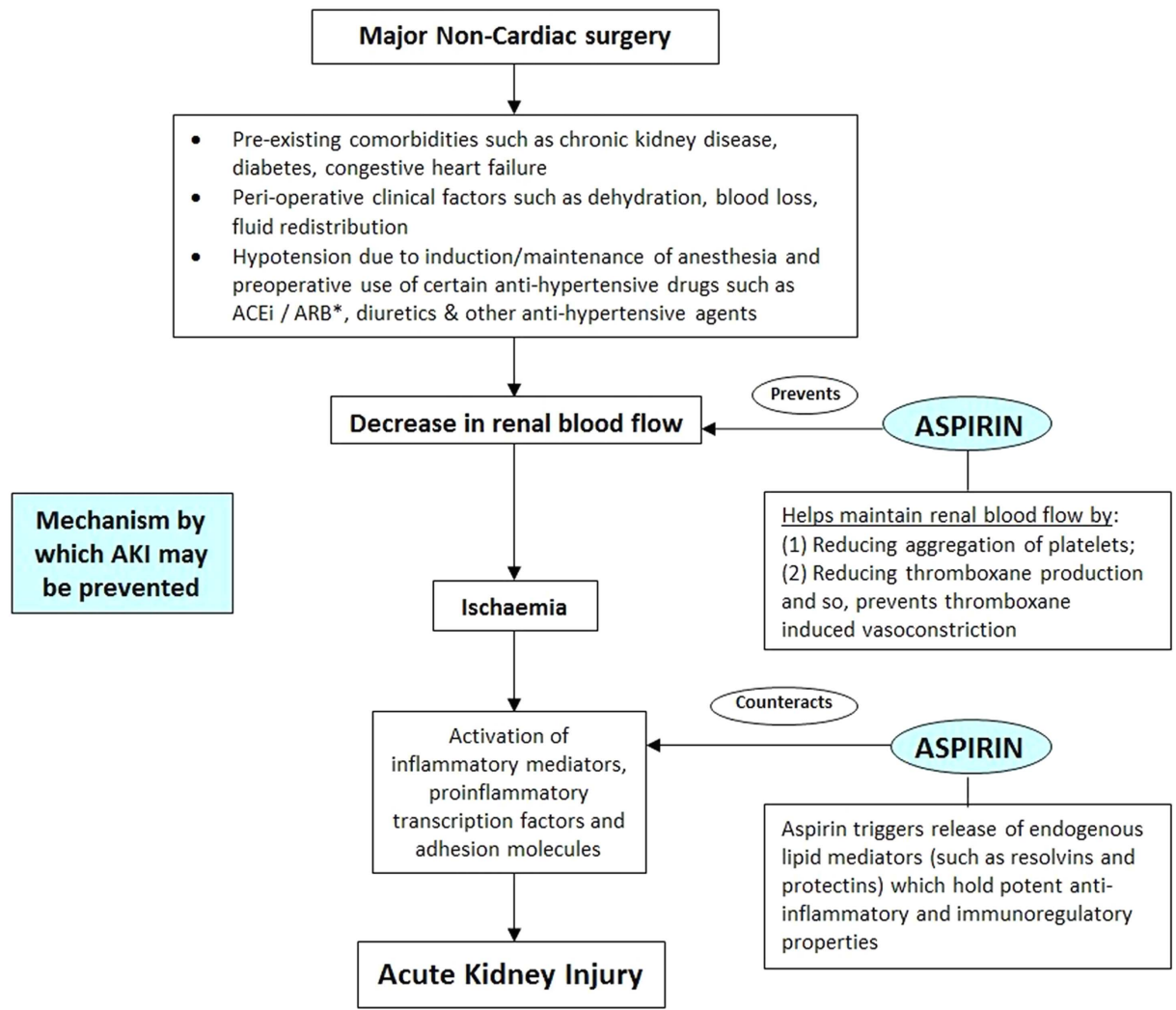

elevated risk of AKI could also manifest from clonidine-induced hypotension).

\section{POISE-2 DATA COLLECTION AND ANALYTIC PLAN}

POISE-2 enrolment began in July 2010, and enrollment in the POISE-2 AKI substudy began in January 2011. To refine the analytic plan, we reviewed POISE-2 data as of April 2013 (when data reconciliation was ongoing and without any knowledge of patient allocation to the assigned treatments).

\section{POISE-2 AKI substudy data collection}

A preoperative serum creatinine value is measured within 6 weeks of surgery. The date of surgery (and not the date of randomisation) is used to identify the start of follow-up in this protocol; the median (IQR) number of days between the date of randomisation and the date of surgery is $0(0-0 ; 95$ th percentile 1 day $)$. After receipt of grant funding to support this substudy, serial perioperative serum creatinine data began to be recorded in consecutive patients enrolled at AKI substudy participating centres (earliest centre to start this process began in January 2011). All centres were encouraged to record a serum creatinine value postoperative days 1,2 and 3 in consecutively enrolled patients (provided the patient was not discharged from hospital). This will help reduce concerns about an ascertainment bias related to AKI (ie, where aspirin vs placebo alters the incidence of another event such as myocardial infarction or bleeding, which in turn influences the likelihood of serum creatinine measurement). We also record all other serum creatinine measurements performed as a part of routine care (and their dates) during the hospital stay. At the time of final analysis, we will examine the number of measurements by treatment group (and the postoperative days of these measurements) to confirm that there is no differential ascertainment of AKI in the two treatment groups.

The median hospital length of stay in POISE-2 is 4 days (IQR 3-7 days). The proportion of patients who die in the operating room or in $48 \mathrm{~h}$ after surgery, which may result in no serum creatinine measurement, is expected to be $<1 \%$ of patients. Less than $10 \%$ of patients are expected to have a missing peak serum creatinine value during the hospital stay, and in such cases, in the absence of receipt of acute dialysis, we will carry forward the prerandomisation serum creatinine value for all analyses (which means the patient will not have developed AKI; see analysis section). Urine output data are, however, not collected in POISE-2 given the difficulties with accurate measurement in the setting of international data collection (and thus a low urine output is not used to define AKI in POISE-2). Receipt of new dialysis for kidney failure is recorded at hospital discharge and at 30 days after surgery.

\section{Longer term kidney function measurement}

In participating centres, at the time of randomisation, patients were invited to enrol in a substudy to record a 
single longer term serum creatinine value, anytime between 3 and 12 months after surgery. A measurement performed at a time when a patient is not acutely ill is valid.

The additional serum creatinine collection (particularly the longer term creatinine measurement) was added to the patient consent requests and received ethical approval at each of the AKI substudy participating sites.

\section{Patient selection}

All patients enrolled in the POISE-2 AKI substudy at the time of randomisation will be included in the final analysis except for the following three reasons: (1) those with endstage renal disease prior to randomisation (expected $<2 \%$ of patients), as the assessment of AKI is no longer relevant (estimated-glomerular filtration rate (eGFR) $<15 \mathrm{~mL} /$ $\min / 1.73 \mathrm{~m}^{2}$ as determined by the CKD-epidemiology collaboration equation, ${ }^{25}$ receipt of chronic dialysis or a baseline serum creatinine $>327 \mu \mathrm{mol} / \mathrm{L}$ (the last exclusion also enables retained patients to have their new-onset AKI staged according to most recent guidelines $\left.{ }^{26}\right)$ ), (2) those missing a pre-randomisation serum creatinine value or missing age or sex (expected in $<3 \%$ of patients) as this is needed to define baseline CKD or subsequent AKI, (3) those who never underwent surgery (expected in $<2 \%$ of patients) as they will not have the opportunity to have any postoperative serum creatinine measurements. We expect to enrol over 6500 eligible patients in the POISE-2 AKI substudy, with a subpopulation of at least 3000 patients who, at the time of randomisation, agree to the longer term creatinine measurement.

\section{Intention to treat}

The intention-to-treat principle will guide all primary analyses, irrespective of whether there is a deviation from the randomly allocated therapy. Currently, $<1.5 \%$ of patients in POISE-2 did not receive the study medication (neither aspirin nor clonidine), $0.1 \%$ of patients received non-study aspirin within $24 \mathrm{~h}$ prior to surgery and $5.2 \%$ received non-study aspirin in the first 3 days after surgery. At the time of final analysis, these results will be reported by the treatment group.

\section{Primary definition of acute kidney injury}

We will use a mixed effects logistic regression model to obtain an estimate of the OR of AKI comparing aspirin to placebo (after testing model assumptions; online supplementary appendix 2). While logistic regression models will be used for hypothesis testing, at the time of final analysis we will preferentially report relative risk reductions of aspirin relative to placebo as this metric is easier to interpret by a clinical audience (and hence we use relative risk reduction nomenclature throughout this protocol appreciating that the logistic regression model produces an OR; the method to derive a relative risk reduction from an OR is presented in online supplementary appendix 3$).{ }^{27}$
AKI will be primarily defined as per recent guidelines as any of the following two criteria: (1) an increase in serum creatinine $\geq 26.5 \mu \mathrm{mol} / \mathrm{L}$ within $48 \mathrm{~h}$ of surgery or (2) an increase in serum creatinine $\geq 50 \%$ from baseline within 7 days of surgery. ${ }^{26}$ For the primary analysis, we will treat centre as a random effect and adjust for the following baseline characteristics: ${ }^{28}$ age (per year), sex, presence of cardiovascular disease (any coronary artery disease, peripheral vascular disease or stroke), presence of diabetes, preoperative eGFR category $(>60 \mathrm{~mL} / \mathrm{min}$ / $1.73 \mathrm{~m}^{2}$ vs $\leq 60 \mathrm{~mL} / \mathrm{min} / 1.73 \mathrm{~m}^{2}$ ), a history of smoking within 2 years of surgery, receipt of urgent or emergency surgery (about $8 \%$ of POISE-2 participants), type of surgery (major vascular surgery, major thoracic surgery, other surgery), chronic use of aspirin therapy and use of the following medications in each of two periods prior to randomisation ( 7 days to $<6 \mathrm{~h}$ prior to surgery, and $\leq 6 \mathrm{~h}$ prior to surgery): COX-2 inhibitor/non-steroidal antiinflammatory drugs/non-COX-2 inhibitor, a statin, an ACE inhibitor/angiotensin receptor blocker/direct renin inhibitor and use of another antihypertensive agent (any of the following: a rate controlling calcium channel blocker, dihydropyridine calcium channel blocker or a $\beta$-blocker). We will also adjust for the random allocation of clonidine (clonidine versus placebo). In patients who underwent surgery but are missing a postoperative serum creatinine value (expected $<10 \%$ of patients), we will impute in its place the prerandomisation serum creatinine value which should provide a more conservative estimate of the intervention effect than the alternative of removing such patients. We expect that $<0.5 \%$ data will be missing for each variable, and if missing we will consider the condition to be absent. We will report the $95 \%$ CI of our estimates, and a two-tailed $p$ value of $\leq 0.05$ will be considered statistically significant. With a sample of at least 6500 patients, we will have over $80 \%$ power to detect at least a $20 \%$ relative risk reduction in AKI with aspirin versus placebo should it exist (anticipated incidence of AKI of $\sim 12 \%$ in the placebo group, two-tailed $\alpha=0.05, \chi^{2}$ test; table 1 ). For completeness and interpretation, in the setting of a significant adjusted relative risk reduction we will also report the unadjusted relative risk reduction (with 95\% $\mathrm{CI}$ ) and the unadjusted absolute risk difference (with 95\% CI). A significant unadjusted absolute risk difference will also be expressed as the 'number needed to treat (NNT)' (1/absolute risk difference; a measure which indicates how many patients need to receive perioperative aspirin to prevent one patient from developing AKI who otherwise would develop AKI if they receive placebo; a lower number indicating a greater benefit of aspirin). The 95\% CI of the NNT is the inverse of the Wald CI for the absolute risk difference. ${ }^{29} 30$

\section{Alternate definitions of AKI}

To determine how robust the AKI results are, we will examine the effect of aspirin versus placebo on alternate definitions of AKI. A two-tailed $p$ value $\leq 0.05$ will be 
Table 1 Incidence of acute kidney injury in POISE-2 and associated statistical power to detect an intervention effect

\begin{tabular}{|c|c|c|c|c|}
\hline & $\begin{array}{l}\text { All patients } \\
(n=4880)^{\star}\end{array}$ & $\begin{array}{l}\text { Patients with a } \\
\text { preoperative } \\
\text { eGFR }>60 \mathrm{~mL} / \mathrm{min} / 1.73 \mathrm{~m}^{2} \\
(\mathrm{n}=3690)\end{array}$ & $\begin{array}{l}\text { Patients with a } \\
\text { preoperative } \\
\text { eGFR } \leq 60 \mathrm{~mL} / \mathrm{min} / 1.73 \mathrm{~m}^{2} \\
(\mathrm{n}=1190)\end{array}$ & $\begin{array}{l}\text { Statistical power to } \\
\text { detect a } 20 \% \text { relative } \\
\text { risk reduction } \\
\text { (6500 patients) }\end{array}$ \\
\hline AKI (primary definition) & $580(11.9 \%)$ & $398(10.8 \%)$ & $182(15.3 \%)$ & $88 \%$ \\
\hline $\begin{array}{l}\text { Alternate definitions } \\
\mathrm{AKI} \text { or death }\end{array}$ & $583(12.0 \%)$ & $399(10.8 \%)$ & $184(15.5 \%)$ & $88 \%$ \\
\hline AKI for at least 2 days & $309(6.3 \%)$ & $190(5.2 \%)$ & $119(10.0 \%)$ & $57 \%$ \\
\hline Stage $2 \mathrm{AKI}$ or more & $138(2.8 \%)$ & $97(2.6 \%)$ & $41(3.5 \%)$ & $32 \%$ \\
\hline
\end{tabular}

considered statistically significant in these analyses if results are concordant with the primary results. The statistical power to detect a $20 \%$ relative risk reduction in each of these outcomes is also presented in table 1. As seen, there is inadequate statistical power to detect a clinically important difference in some outcomes (such as stage $2 \mathrm{AKI}$ ); these outcomes will be reported given their clinical significance and we will visually compare the point estimates and $95 \%$ CI of the relative risk reduction for each definition of AKI.

- AKI or death: A composite outcome of either the primary AKI definition or death within $48 \mathrm{~h}$ of surgery. This is to account for the potential impact that early deaths may have on the ascertainment of AKI.

- AKI for at least 2 days: Defined by evidence of a $\geq 50 \%$ or $\mathrm{a} \geq 26.5 \mu \mathrm{mol} / \mathrm{L}$ increase in postoperative serum creatinine from the preoperative value, evident on at least two different days within 7 days of surgery. While the magnitude of the peak change in serum creatinine defines the stage of AKI in recent guidelines, a longer duration of AKI is also associated with poorer outcomes. $^{31}$

- Stage 2 AKI or more. Defined as any of the following three criteria-(1) postoperative percent increase in serum creatinine $\geq 100 \%$ from the preoperative value within 7 days of surgery, (2) increase in postoperative serum creatinine to an absolute value $\geq 353.6 \mu \mathrm{mol} / \mathrm{L}$ within 7 days or (3) receipt of acute dialysis within 30 days.

In addition to dichotomous outcomes, we will use a linear regression model to compare the two groups in the outcome of percent change in serum creatinine ( ( peak postoperative serum creatinine (within 7 days of surgery)-preoperative serum creatinine)/preoperative serum creatinine), adjusting for the variables described in the primary outcome analysis (assuming model assumptions are not violated; testing presented in online supplementary appendix 2). We will report the result as the average difference in per cent change in serum creatinine between the two treatment groups with $95 \%$ CI.

\section{Subgroup analyses: presence of preoperative CKD and} chronic aspirin use

We will use an interaction term in a mixed effects logistic regression model where centre is treated as a random effect to determine if the adjusted OR of AKI comparing aspirin to placebo differs in those with and without CKD (assuming model assumptions are not violated; testing presented in online supplementary appendix 2). We will interpret a two-tailed $\mathrm{p}$ value $\leq 0.05$ as statistically significant. With 6500 patients, we will have over $80 \%$ power to detect a $45 \%$ lower OR of AKI with treatment for patients with and without CKD (an estimate derived from our simulations; in another recent large trial of coronary artery bypass graft surgery performed with and without a bypass pump, the observed percent decrease in the OR of AKI was $40 \%$ lower in patients with CKD compared to those without CKD) ( $p$ value for interaction 0.01). Similar to techniques used for the CKD subgroup, we will examine subgroups defined by the presence of preoperative chronic aspirin use (which was a stratification factor used in the randomisation-aspirin initiation and aspirin continuation strata). Similarly, we will have over $80 \%$ power to detect a $40 \%$ lower OR of AKI with treatment for patients in the continuation stratum compared to the initiation stratum. An increased risk of thrombosis after aspirin discontinuation has been observed in some human studies, recognising that the biology is complicated. ${ }^{18}$

\section{Additional analyses}

Longer term kidney function loss after surgery

This analysis will focus on those patients who, at the time of surgery, consent to a single serum creatinine measurement between 3 and 12 months after surgery. For our main analysis, we will use linear regression adjusting for prior listed covariates to compare the absolute change in eGFR between the aspirin and placebo groups, reporting the mean and 95\% CI (assuming model assumptions are not violated, online supplementary appendix 2). We will carry forward the 
prerandomisation serum creatinine value for patients with a missing longer term value after surgery (for reasons of death or missing measurement) and impute an eGFR value of $5 \mathrm{~mL} / \mathrm{min} / 1.73 \mathrm{~m}^{2}$ for the long-term measurement for any patient who developed end-stage renal disease ( $\geq 3$ months of continuous dialysis), or who died shortly after receipt of acute dialysis for severe AKI. A sample of 3000 patients will allow a $5 \mathrm{~mL} / \mathrm{min} /$ $1.73 \mathrm{~m}^{2}$ or more difference to be detected between the two groups with over $80 \%$ statistical power (two-tailed $\alpha=0.05$, independent samples $t$ test, expected $S D=40$ ). As observed in the setting of another international trial of coronary artery bypass surgery performed with and without a cardiopulmonary bypass pump, we do not expect to demonstrate that perioperative aspirin versus placebo alters this outcome, even in the situation where we demonstrate aspirin versus placebo reduces the risk of postoperative AKI (which may relate to our study methods or the limited impact of any observed AKI risk reduction on longer term kidney function).

\section{Postoperative cardiac events}

Acute cardiac events are inextricably linked to AKI events and both events often occur together in the nonoperative setting. In the setting where aspirin versus placebo reduces the risk of AKI, we will also examine cardiac events in the analysis and report how frequently both co-occur.

\section{A higher risk of AKI with aspirin}

Although we hypothesise that aspirin use will prevent perioperative AKI, our analysis may also elucidate AKI harm from aspirin if it exists. Although nephrotoxicity from non-steroidal anti-inflammatory agents is well appreciated (through the inhibition of intrarenal prostaglandin), aspirin doses of $100 \mathrm{mg} /$ day, as used in POISE-2, are unlikely to be nephrotoxic. ${ }^{32}$ However, bleeding with aspirin is an important concern which can cause hypotension and AKI. ${ }^{73}$ Similarly, blood transfusions may directly predispose to AKI, possibly through some haemolysis. ${ }^{34}$ If we observe that aspirin versus placebo increases the risk of AKI, then we will examine bleeding events and AKI and report how frequently they both co-occur.

\section{Exclusion of major urological surgeries}

POISE-2 records surgical types in categories, and one of these categories is major urological or gynaecological surgery (about $13 \%$ of patients enrolled in POISE-2). This category includes the procedure of nephrectomy. Because postoperative changes in serum creatinine in the context of partial or complete nephrectomy can occur for reasons other than AKI, in an additional analysis we will exclude this category of surgery to confirm that the results on the remaining sample are no different than the primary results.

\section{Effects of clonidine on AKI}

While our prespecified hypotheses focus on aspirin, it is conceivable that clonidine may reduce the risk of AKI as supported by prior animal and human studies. ${ }^{19-24}$ The analytic techniques for the assessment of clonidine on AKI are as specified for aspirin, with only minor adjustments (ie, adjustment for the random allocation to aspirin and no prespecified subgroup analysis for chronic aspirin use). If a greater risk of AKI is observed with clonidine versus placebo, then the blood pressure data collected in POISE-2 will be reviewed to determine if this potentially explains the observed effect.

\section{RECOGNISED LIMITATIONS}

There are some limitations to our protocol.

\section{Errors with serum creatinine as a measure of kidney function}

In POISE-2, the preoperative serum creatinine is recorded only once in the 6 weeks prior to surgery (where patients may be undergoing elective, urgent or emergent surgery). It would be preferable to have at least two baseline serum creatinine values on all POISE-2 patients, separated by at least 3 months, to more accurately define the presence of CKD. Also, in POISE-2, there is no knowledge of potential serum creatinine changes close to the time of surgery (particularly in the case of urgent/emergent surgery; $8 \%$ of POISE-2 patients), which may mean that the preoperative serum creatinine value is not in a steady state. All of these considerations increase 'noise' related to the baseline serum creatinine measurement, and reduce the ability to detect postoperative changes in the serum creatinineto partly address this, we are adjusting for a definition of urgent/emergent surgery in the primary analysis of covariance models. Also, in an additional analysis, we will exclude patients undergoing urgent/elective surgery to confirm that the findings are robust in the remaining patients undergoing elective surgery.

Worldwide, over the last decade, there have been efforts to better standardise the serum creatinine assay. It seems likely (although not documented in POISE-2) that most preoperative and postoperative serum creatinine measurements prior to hospital discharge would be performed in the same laboratory, and most measurements are performed within 2 months of each other. This reduces concerns about interlaboratory and intralaboratory variability (drift) in the serum creatinine measurement. However, the same cannot be said for the longer term serum creatinine measurement. As with the preoperative value, multiple serial measures of serum creatinine over the year (and longer) were desired, but not possible to obtain in POISE-2. Also, POISE-2 has no measures of baseline or follow-up proteinuria, which are now featured in recent CKD staging systems. ${ }^{35}$ However, the randomisation and analysis of this substudy is stratified by centre, which should result in similar 
measurement errors within a centre for the aspirin and placebo groups.

\section{Limited statistical power for the most clinically important kidney outcomes}

Stage 3 AKI with receipt of acute dialysis, and long-term end-stage renal disease are the most clinically important kidney outcomes. However, there will be too few events for adequate statistical power to detect these outcomes in POISE-2. We focus on mild to moderate AKI (defined by thresholds of changes in serum creatinine). While mild-to-moderate AKI is the outcome used in virtually all prior AKI prevention trials, we recognise that it is a surrogate outcome that may not directly impact how a patient feels, functions or survives. Similarly, the longer term outcome of between-group difference in change in eGFR is also a surrogate outcome, and concerns about its validity as a measure of kidney disease progression have been raised. ${ }^{36}$ For this reason, the overall POISE-2 trial results (which focus on non-kidney outcomes), rather than the results of this AKI protocol, should be the primary information used to inform decisions about whether the tested interventions should be adopted as a standard in surgical care.

\section{CONCLUSIONS}

Strengths of this POISE-2 AKI protocol and the overall POISE-2 trial are generalisable estimates derived from patients recruited in an international context using rigorous randomised trial methodology (eg, concealed allocation, placebo-controlled trial, blinded central adjudication of outcomes). In this report, we have judiciously prespecified the main questions and analytic protocol that will be used to test relevant AKI hypotheses in the POISE-2 AKI substudy. This protocol will help minimise the chance of spurious post hoc assertions of effect, so that the AKI results from this large international are robust and believable.

\author{
Author affiliations \\ ${ }^{1}$ Western University/London Health Sciences Centre, London, Canada \\ ${ }^{2}$ Cleveland Clinic, Cleveland, Ohio, USA \\ ${ }^{3}$ University of Waterloo, Waterloo, Canada \\ ${ }^{4}$ Population Health Research Institute/McMaster University/Hamilton Health \\ Sciences/St Joseph's Healthcare, Hamilton, Canada \\ ${ }^{5}$ Yale University, Connecticut, USA \\ ${ }^{6}$ Hospital de la Santa Creu i Sant Pau, Barcelona, Spain \\ ${ }^{7}$ Fundación Oftalmológica de Santander (FOSCAL), Bucaramanga, Colombia \\ ${ }^{8}$ Copenhagen University Hospital Herlev, Herlev, Denmark \\ ${ }^{9}$ The Chinese University of Hong Kong, Hong Kong Special Administrative \\ Region, China \\ ${ }^{10}$ Clinica Santa Maria, Santiago, Chile \\ ${ }^{11}$ Kingston General Hospital, Kingston, Canada \\ ${ }^{12}$ Hospital Vall d'Hebron, Barcelona, Spain \\ ${ }^{13}$ Shifa International Hospitals Limited, Islamabad, Pakistan \\ ${ }^{14} \mathrm{CHU}$ Brugmann, Brussels, Belgium \\ ${ }^{15}$ San Raffaele Scientific Institute, Milan, Italy \\ ${ }^{16}$ Vejle Hospital, Vejle, Denmark \\ ${ }^{17}$ Hospital Nacional Cayetano Heredia, Lima, Peru \\ ${ }^{18}$ Monash University, Melbourne, Australia \\ ${ }^{19}$ University of North Carolina Medical School, Chapel Hill, North Carolina, \\ USA
}

${ }^{20}$ Vienna General Hospital/Medical University of Vienna, Vienna, Austria

${ }^{21}$ Fundación Cardioinfantil (FCl), Bogotá, Colombia

${ }^{22}$ Royal Adelaide Hospital, Adelaide, Australia

${ }^{23}$ Nelson R Mandela School of Medicine, Durban, South Africa

${ }^{24}$ The Ohio State University Medical Center, Columbus, Ohio, USA

${ }^{25}$ University of Manitoba, Winnipeg, Canada

${ }^{26}$ University of Texas-MD Anderson Cancer Center, Houston, Texas, USA

${ }^{27}$ University of Toronto, Toronto, Canada

${ }^{28}$ Mahatma Gandhi Institute of Medical Sciences, Wardha, Maharashtra, India

${ }^{29}$ Royal Melbourne Hospital, Melbourne, Australia

Acknowledgements The authors would like to thank Dr Mitesh Shah for his contributions when submitting the associated Canadian Institutes of Health Research grant, which included a background literature review.

Contributors All the authors contributed to the conception and design, as well as the acquisition, analysis and interpretation of the data; drafting of the protocol or revising it critically for important intellectual content and final approval of the version to be published.

Funding The POISE-2 trial and the AKI substudy are funded by two grants from the Canadian Institutes of Health Research (application number of the AKI substudy: 259720). General support for POISE-2 was also provided by the Australian National Health and Medical Research Council and the Spanish Ministry of Health and Social Policy. Boehringer Ingelheim provided the clonidine study drug and some funding, and Bayer Schering Pharma provided the aspirin study drug. The sponsors of POISE-2 and the AKI substudy had no role in the design and conduct of the study; collection, management, analysis and interpretation of the data; preparation, review or approval of this protocol paper; and decision to submit this protocol manuscript for publication.

Competing interests None.

Ethics approval The REB of each participating site; 82 centres in 21 countries.

Provenance and peer review Not commissioned; internally peer reviewed.

Open Access This is an Open Access article distributed in accordance with the Creative Commons Attribution Non Commercial (CC BY-NC 3.0) license, which permits others to distribute, remix, adapt, build upon this work noncommercially, and license their derivative works on different terms, provided the original work is properly cited and the use is non-commercial. See: http:// creativecommons.org/licenses/by-nc/3.0/

\section{REFERENCES}

1. Weiser TG, Regenbogen SE, Thompson KD, et al. An estimation of the global volume of surgery: a modelling strategy based on available data. Lancet 2008;372:139-44.

2. Abelha FJ, Botelho M, Fernandes V, et al. Determinants of postoperative acute kidney injury. Crit Care 2009;13:R79.

3. Chertow GM, Burdick E, Honour M, et al. Acute kidney injury, mortality, length of stay, and costs in hospitalized patients. J Am Soc Nephrol 2005;16:3365-70.

4. Ricci Z, Cruz D, Ronco C. The RIFLE criteria and mortality in acute kidney injury: a systematic review. Kidney Int 2008;73:538-46.

5. Coca SG, Yusuf B, Shlipak MG, et al. Long-term risk of mortality and other adverse outcomes after acute kidney injury: a systematic review and meta-analysis. Am J Kidney Dis 2009;53:961-73.

6. Landoni G, Bove T, Szekely A, et al. Reducing mortality in acute kidney injury patients: systematic review and international web-based survey. J Cardiothorac Vasc Anesth 2013;27:1384-98.

7. Gerrah R, Izhar U. Beneficial effect of aspirin on renal function post-cardiopulmonary bypass. Asian Cardiovasc Thorac Ann 2003;11:304-8.

8. Gerrah R, Ehrlich S, Tshori S, et al. Beneficial effect of aspirin on renal function in patients with renal insufficiency postcardiac surgery. $J$ Cardiovasc Surg (Torino) 2004;45:545-50.

9. Mangano DT. Aspirin and mortality from coronary bypass surgery. N Engl J Med 2002;347:1309-17.

10. Serhan CN, Arita M, Hong S, et al. Resolvins, docosatrienes, and neuroprotectins, novel omega-3-derived mediators, and their endogenous aspirin-triggered epimers. Lipids 2004;39:1125-32. 
11. Serhan CN, Gotlinger K, Hong S, et al. Resolvins, docosatrienes, and neuroprotectins, novel omega-3-derived mediators, and their aspirin-triggered endogenous epimers: an overview of their protective roles in catabasis. Prostaglandins Other Lipid Mediat 2004;73:155-72.

12. Serhan CN, Chiang N, Van Dyke TE. Resolving inflammation: dual anti-inflammatory and pro-resolution lipid mediators. Nat Rev Immunol 2008;8:349-61.

13. Serhan CN. Resolution phase of inflammation: novel endogenous anti-inflammatory and proresolving lipid mediators and pathways. Annu Rev Immunol 2007;25:101-37.

14. Duffield JS, Hong S, Vaidya VS, et al. Resolvin D series and protectin D1 mitigate acute kidney injury. J Immunol 2006;177:5902-11.

15. Cao L, Young N, Liu H, et al. Preoperative aspirin use and outcomes in cardiac surgery patients. Ann Surg 2012;255:399-404.

16. Rosner $\mathrm{MH}$, Okusa MD. Acute kidney injury associated with cardiac surgery. Clin J Am Soc Nephrol 2006;1:19-32.

17. Devereaux PJ. http://clinicaltrials.gov/ct2/show/NCT01082874? term=POISE-2\&rank=1. (last accessed 16 Jan 2014), 2014.

18. Doutremepuich $\mathrm{C}$, Aguejouf $\mathrm{O}$, Desplat V, et al. Aspirin discontinuation syndromes: clinical implications of basic research studies. Am J Cardiovasc Drugs 2013;13:377-84.

19. Kulka PJ, Tryba M, Zenz M. Preoperative alpha2-adrenergic receptor agonists prevent the deterioration of renal function after cardiac surgery: results of a randomized, controlled trial. Crit Care Med 1996;24:947-52.

20. Myles PS, Hunt JO, Holdgaard HO, et al. Clonidine and cardiac surgery: haemodynamic and metabolic effects, myocardial ischaemia and recovery. Anaesth Intensive Care 1999;27:137-47.

21. Billings FT, Chen SW, Kim M, et al. Alpha2-Adrenergic agonists protect against radiocontrast-induced nephropathy in mice. $A m \mathrm{~J}$ Physiol Renal Physiol 2008;295:F741-8.

22. Eknoyan G, Dobyan DC, Senekjian HO, et al. Protective effect of oral clonidine in the prophylaxis and therapy of mercuric chlorideinduced acute renal failure in the rat. $J$ Lab Clin Med 1983;102:699-713.
23. Solez K, Racusen LC, Whelton A. Glomerular epithelial cell changes in early postischemic acute renal failure in rabbits and man. $A m J$ Pathol 1981;103:163-73.

24. Ideura $\mathrm{T}$, Solez $\mathrm{K}, \mathrm{Heptinstall} \mathrm{RH}$. The effect of clonidine on tubular obstruction in postischemic acute renal failure in the rabbit demonstrated by microradiography and microdissection. Am J Pathol 1980;98:123-50.

25. Levey AS, Stevens LA, Schmid CH, et al. A new equation to estimate glomerular filtration rate. Ann Intern Med 2009;150:604-12.

26. Kidney Disease: Improving Global Outcomes (KDIGO) Acute Kidney Injury Work Group. KDIGO clinical practice guidelines for acute kidney injury. Kidney Int 2012;2:1-138.

27. Austin PC. Absolute risk reductions, relative risks, relative risk reductions, and numbers needed to treat can be obtained from a logistic regression model. J Clin Epidemiol 2010;63:2-6.

28. Kahan BC, Morris TP. Adjusting for multiple prognostic factors in the analysis of randomised trials. BMC Med Res Methodol 2013;13:99.

29. Altman DG. Confidence intervals for the number needed to treat. BMJ 1998;317:1309-12.

30. Bender R. Calculating confidence intervals for the number needed to treat. Control Clin Trials 2001;22:102-10.

31. Brown JR, Kramer RS, Coca SG, et al. Duration of acute kidney injury impacts long-term survival after cardiac surgery. Ann Thorac Surg 2010;90:1142-8.

32. Juhlin $T$, Jonsson BA, Hoglund P. Renal effects of aspirin are clearly dose-dependent and are of clinical importance from a dose of 160 mg. Eur J Heart Fail 2008;10:892-8.

33. Acedillo RR, Shah M, Devereaux PJ, et al. The risk of perioperative bleeding in patients with chronic kidney disease: a systematic review and meta-analysis. Ann Surg 2013;258:901-13.

34. Vellinga S, Verbrugghe W, De PR, et al. Identification of modifiable risk factors for acute kidney injury after cardiac surgery. Neth $\mathrm{J}$ Med 2012;70:450-4.

35. Stevens PE, Levin A. Evaluation and management of chronic kidney disease: synopsis of the kidney disease: improving global outcomes 2012 clinical practice guideline. Ann Intern Med 2013;158:825-30.

36. Gaspari F, Ruggenenti P, Porrini E, et al. The GFR and GFR decline cannot be accurately estimated in type 2 diabetics. Kidney Int 2013;84:164-73. 